\title{
Erratum to: Field-effect at electrical contacts to two-dimensional materials
}

Yao Guo ${ }^{1}(\bowtie)$, Yan Sun ${ }^{1}$, Alvin Tang ${ }^{2}$, Ching-Hua Wang ${ }^{2}$, Yanqing Zhao ${ }^{1}$, Mengmeng Bai ${ }^{1}$, Shuting Xu', Zheqi Xu ${ }^{1}$, Tao Tang ${ }^{3}$, Sheng Wang ${ }^{4}$, Chenguang Qiu ${ }^{4}$, Kang Xu${ }^{5}$, Xubiao Peng ${ }^{1}$, Junfeng Han ${ }^{1}$, Eric Pop ${ }^{2}$, and Yang Chai ${ }^{5}(\square)$

${ }^{1}$ School of Physics, Beijing Institute of Technology, Beijing 100081, China

${ }^{2}$ Department of Electrical Engineering and Stanford SystemX Alliance, Stanford University, Stanford, CA 94305, USA

${ }^{3}$ Advanced Manufacturing EDA Co., Ltd., Shanghai 201204, China

${ }^{4}$ Key Laboratory for the Physics and Chemistry of Nanodevices, Department of Electronics, Peking University, Beijing 100871, China

${ }^{5}$ Department of Applied Physics, The Hong Kong Polytechnic University, Hong Kong, China

(C) Tsinghua University Press and Springer-Verlag GmbH Germany, part of Springer Nature 2021

Received: 6 April 2021 / Revised: 24 May 2021 / Accepted: 26 May 2021

\section{Erratum to}

Nano Research

https://doi.org/10.1007/s12274-021-3670-y

The article Field-effect at electrical contacts to two-dimensional materials, written by Yao Guo et al., was erroneously originally published electronically on the publisher's internet portal (currently SpringerLink) on 28 July 2021 with Ref. [27] and Table of contents.

\section{Instead of}

[27] McClellan, C. J.; Yalon, E.; Smithe, K. K. H.; Suryavanshi, S. V.; Pop, E. Effective n-type doping of monolayer MoS2 by AlOx. In Proceedings of the 2017 75th Annual Device Research Conference, South Bend, 2017, pp 1-2.

\section{It should read}

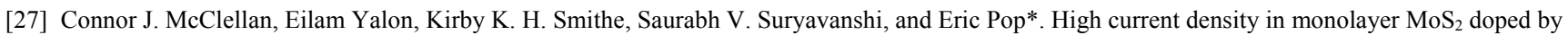
$\mathrm{AlO}_{x}$. ACS Nano 2021, 15, 1587-1596.

Instead of

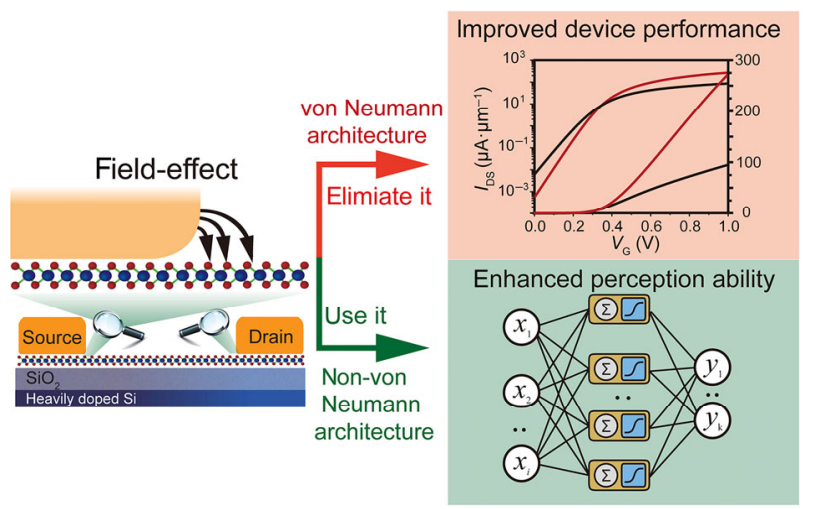

It should read

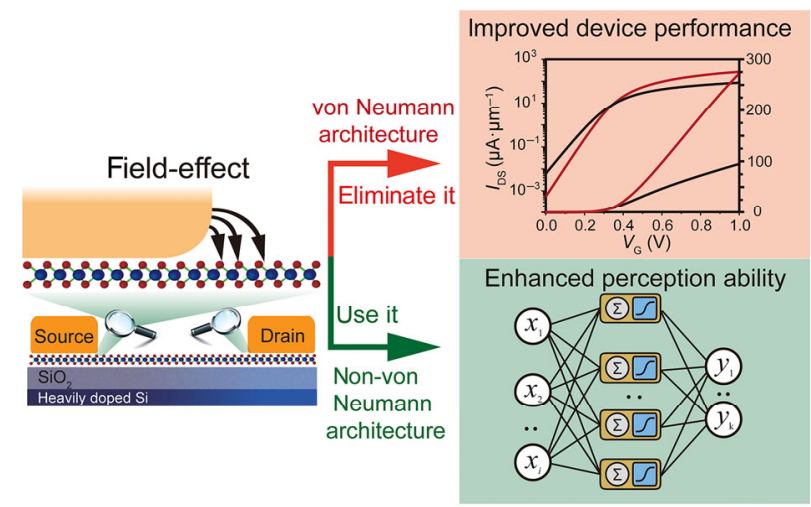

The online version of the original article can be found at https://doi.org/10.1007/s12274-021-3670-y 